East African Medical Journal Vol. 86 No. 5 May 2009

NON CONVERSION OF SPUTUM SMEARS IN NEW SMEAR POSITIVE PULMONARY TUBERCULOSIS PATIENTS IN YAOUNDÉ, CAMEROON

C. Kuaban, MD, CES, MPH, Department of Internal Medicine, Faculty of Medicine and Biomedical Sciences, University of Yaoundé.1/ Hôpital Jamot, Yaoundé, Cameroon, R. Bame, MD, L. Mouangue, MD, S. Djella, MD and C. Yomgni, SRN, Department of Chest Medicine, Hôpital Jamot, Yaoundé, Cameroon

Request for reprints to: Prof. C. Kuaban, Department of Chest Medicine, Hôpital Jamot, B.P 4021 Yaoundé, Cameroon

\title{
NON CONVERSION OF SPUTUM SMEARS IN NEW SMEAR POSITIVE PULMONARY TUBERCULOSIS PATIENTS IN YAOUNDÉ, CAMEROON
}

\author{
C. KUABAN, R. BAME, L. MOUANGUE, S. DJELLA and C. YOMGNI
}

\begin{abstract}
Objectives: To identify clinical, radiological and microbiological factors associated with the non conversion of sputum smears in new smear positive cases of pulmonary tuberculosis after two months of treatment and to evaluate the influence of non-smear conversion on treatment outcomes.

Design: A prospective cohort study.

Setting: Tuberculosis centre of Hôpital Jamot in Yaoundé- Cameroon from April 2006 to September 2007.

Subjects: A total of 413 patients were studied.

Main outcome measures: Sputum smear status at two months of treatment, favourable treatment outcome (cured, treatment completed), unfavourable treatment outcome (death, treatment failure, default from treatment) and transferred out.

Results: A total of 413 patients were studied; There were $234(56.8 \%)$ males and 178 $(43.2 \%)$ females with a mean age of 33 years (range 9.80 years). Sputum smears did not convert in $55(13.4 \%)$ patients at the end of two months of treatment. Logistic regression analysis showed that age above or equal to 40 years $(\mathrm{OR}=2.716,95 \% \mathrm{CI}: 1.412-5.223, \mathrm{p}=$ $0.003)$, and a bacillary load of $3+$ on pre-treatment sputum smears $(\mathrm{OR}=1.955 ; 95 \% \mathrm{CI}$ : 1.039-3.68, $\mathrm{p}=0.037$ ) were significantly associated with non conversion of sputum smears at the end of two months of treatment. Persistent positive smears at the end of two months of treatment were significantly associated with unfavourable treatment outcomes $(p=0.025)$ especially default during the course of treatment.

Conclusion: In Yaoundé, Cameroon, non conversion of positive sputum smears in new patients with pulmonary tuberculosis at the end of two months of treatment is associated with an unfavourable outcome particularly defaulting later in the course of treatment. Non conversion of sputum smears at two months of treatment is significantly associated with age above or equal to 40 years and the presence of numerous bacilli (3+) on pre-treatment sputum smears. Patients with these factors who do not smear convert after two months of treatment should be given a fully supervised treatment for the entire duration of therapy so as to prevent in particular treatment default.
\end{abstract}

\section{INTRODUCTION}

The International Union Against Tuberculosis and Lung Disease (IUATLD) and the World Health Organisation (WHO), both emphasize the necessity of monitoring treatment by bacteriologic follow-up examination in sputum smear positive tuberculosis patients $(1,2)$. The results of the follow-upexamination at the end of the two month intensive phase of treatment determines whether the therapeutic regimen can be switched to the continuation phase or whether the patient should be given an additional month of intensive phase treatment. Despite the use of this strategy, several studies have shown that among sputum smear positive pulmonary tuberculosis patients, persistent sputum positivity at the end of two months of antituberculosis treatment can predict adverse outcomes in terms of increased failure and / or relapse rates as well as default from treatment (3-8). Knowledge of factors associated with persistent sputum positivity at the end of two months of antituberculosis therapy may therefore be 
useful to clinicians to better manage their patients and improve outcomes.

The aim of this study was to identify simple determinants of non conversion of sputum smears in new smear positive cases of pulmonary tuberculosis and evaluate its influence on treatment outcome in Yaoundé, Cameroon.

\section{MATERIALS AND METHODS}

Study setting: The study was undertaken from April 2006 to September 2007 at the tuberculosis centre of Hôpital Jamot, the sole referral and tuberculosis treatment facility for Yaoundé and surrounding areas. In this centre, a diagnosis of smear positive pulmonary tuberculosis is made on the basis of a suggestive clinical history and the presence of acid-fast bacilli (AFB) on at least two of the three sputum samples submitted on two consecutive days by the patient for microscopic examination after staining by the Ziehl Neelsen's technique. Cultures for mycobacteria are hardly ever done.

All newly diagnosed and previously untreated patients with sputum smear positive pulmonary tuberculosis are hospitalised during the intensive phase of treatment which lasts for two months. The total duration of treatment is six months. It consists of a two month intensive phase of daily rifampicin $(\mathrm{R})$, isoniazid $(\mathrm{H})$ pyrazinamide $(\mathrm{Z})$ and ethambutol (E), followed by a four month continuation phase of daily rifampicin and isoniazid on out-patient basis. Treatment in the intensive phase is administered under direct supervision of the health personnel while compliance to treatmentduring the continuation phase is assessed by monthly return for drug collection.

During the six month treatment period, the patient's sputum is checked thrice for the presence or absence of AFB by direct microscopic examination. This is done at the end of the two monthintensive phase and at the end of the fifth and sixth month of treatment. In patients whose sputum smears remain positive at the end of the two month phase of treatment, an additional month of intensive phase treatment is given followed by the continuation phase. The outcome of patients at the end of the six months of treatment is recorded into one of the following six mutually exclusive categories according to the IUATLD and WHO $(1,2)$ :

i) Cured:Treatmentcompleted with anegativesputum smear in the last month of treatment and on at least one previous occasion;

ii) Treatment completed: Patient who has completed treatment but does not meet the criteria to be classified as a cure or failure;

iii) Treatment failure: Patient who is sputum smear positive at five months or later during treatment;

iv) Died: Patient who dies for any reason during the course of treatment; v) Defaulted:Patient whose treatment was interrupted for two consecutive months or more:

vi) Transferred out: Patient who has been transferred to another recording and reporting unit and for whom treatment outcome is known.

Methods: A prospective cohort of all patients consecutively admitted to the centre with sputum smear positive and previously untreated pulmonary tuberculosis was studied. After obtaining informed consent from each patient on inclusion, information was got from each patient on age, sex, past history of BCG vaccination, associated history of diabetes mellitus, alcohol and tobacco use as well as the duration of symptoms before the diagnosis of pulmonary tuberculosis. An admission chest X-ray for each patient was reviewed and interpreted. Each chest radiograph was divided into six zones by eye and the presence or absence of parenchymal disease was recorded for each zone. The presence or absence of cavities as well as their number when present was also noted. A blood sample was collected from each patient for HIV serology testing after counselling. HIV antibodies were detected using two rapid tests: Determine $^{\mathrm{R}}$ HIV1/2 (Abbot Laboratories, Tokyo, Japan) and Immuno Combr II HIV1 and 2 BiSpot (Orgenics, Courbevoie, France). When the two tests were positive, the patient was considered positive for HIV. In case of discordant results, a confirmation test was carried out by the Western blot technique (New Lav blot Sanofi Diagnostics, Pasteur). The result of the sputum smear of each patient with the highestbacillary load of the three examined for diagnosis measured by the semi-quantitative method in use in tuberculosis control programmes (ie $1+, 2+, 3+)$ (1) was extracted from the medical file and recorded. Each patient was placed on the standard six month antituberculosis treatment regimen (2 RHEZ / 4RH) for two months in hospital. At the end of this intensive phase of treatment a sputum smear examination was done for each one of them. The absence or presence of AFB on the smear of each patient was noted. Patients whose sputum smears remained positive at the end of this initial intensive phase of treatment were given an additional month of intensive phase therapy followed by a three month continuation phase of treatment. Patients whose sputum smears became negative were immediately switched on to the four month continuation phase of treatment. Sputum smears of each patient were again examined for AFB at the end of the fifth and sixth month of treatment. Outcomes of treatment for all patients were then recorded accordingly at the end of six months of therapy. Patients were defined as having a favourable outcome if they were declared cured or had completed treatment. Patients were defined as having an unfavourable outcome if they had failed therapy, defaulted or died.

Statistical analysis: In order to determine factors 
associated with persistent positive sputum smears after two months of treatment, variables of patients with persistent positive sputum smears were compared to those of patients whose smears had become negative. Chi square or Fisher's exact test was used to compare proportions. Logistic regression analysis was performed using variables found to be significantly associated with persistent positive sputum smears at two months of treatment to identify factors which were independently and significantly associated with it. The final treatment outcomes of patients with respect to their sputum smear conversion status at two months of treatment were compared using the chi square test.

\section{RESULTS}

A total of 436 patients were enrolled in the study. Twenty three $(5.5 \%)$ of these 436 patients were excluded from analysis because they either absconded, died or were transferred out of the tuberculosis centre before the end of the two month intensive phase of treatment. Among the remaining 413 patients who completed the initial phase of treatment and had sputum smears controlled for the presence or absence of AFB, there were $234(56.8 \%)$ males and $178(43.2 \%)$ females with a mean age of 33 years (range $9-80$ years). One hundred and twenty one $(29.3 \%)$ of the total 413 patients were HIV seropositive. Fifty five (13.4\%) of the 413 patients were sputum smear positive at the end of two months of treatment.

Table 1 compares the socio-demographic and clinical characteristics of patients with and without persistent sputum smear positivity at the end of two months of treatment. Table 2 compares various chest radiological and microbiological aspects of patients with and without persistent sputum smear positivity at the end of two months of antituberculosis treatment. This univariate analysis showed that among the demographic and clinical factors, age above or equal to 40 years, the male sex, alcohol and tobacco use were significantly associated with persistent sputum smear positivity at the end of two months of treatment (Table 1). Among the radiological and microbiologic factors, chest lesions involving more than two zones and a bacillary load of 3 + were found to be significant, while a positive HIV serostatus had a borderline significance (Table 2). On the multivariate level (Table 3 ) including only these variables significantly associated with persistent sputum smear positivity at the end of two months of treatment on univariate analysis, it was found that age above or equal to 40 years $(\mathrm{OR}=2.716,95 \% \mathrm{CI}$; 1.412- 5.223, $\mathrm{p}=0.003)$ and a bacillary load of $3+$ on initial sputum smear examination $(\mathrm{OR}=1.955 ; 95 \% \mathrm{CI}: 1.039-3.68 \mathrm{p}=$ $0.037)$ were significantly associated with it.

Table 1

Comparison of demographic and clinical characteristics of patients with and without persistent sputum positivity after two months of antituberculosis treatment

\begin{tabular}{|c|c|c|c|}
\hline Characteristic & \multicolumn{2}{|c|}{ Sputum status after two months of treatment } & P-value \\
\hline \multicolumn{4}{|l|}{ Age (years) } \\
\hline$<40$ & 77.7 & 47.3 & \\
\hline$\geq 40$ & 22.3 & 52.7 & $1.9 \times 10^{-6}$ \\
\hline \multicolumn{4}{|l|}{ Sex } \\
\hline Male & 54.2 & 74.5 & \\
\hline Female & 45.8 & 25.5 & 0.004 \\
\hline \multicolumn{4}{|l|}{ BCG } \\
\hline Yes & 62.6 & 69.1 & \\
\hline No & 37.4 & 30.9 & 0.34 \\
\hline \multicolumn{4}{|l|}{ Diabetes } \\
\hline Yes & 02.2 & 0.0 & \\
\hline No & 97.8 & 100.0 & 0.26 \\
\hline \multicolumn{4}{|l|}{ Alcohol use } \\
\hline Yes & 30.7 & 58.2 & \\
\hline No & 69.3 & 41.8 & $6.6 \times 10^{-5}$ \\
\hline \multicolumn{4}{|l|}{ Tobacco use } \\
\hline Yes & 22.9 & 41.8 & \\
\hline No & 77.1 & 58.2 & 0.003 \\
\hline \multicolumn{4}{|c|}{ Delay before diagnosis } \\
\hline$<1$ month & 29.1 & 18.2 & \\
\hline$\geq 1$ month & 70.9 & 81.8 & 0.093 \\
\hline
\end{tabular}




\section{Table 2}

Comparison of chest radiological and microbiological aspects of patients with and without persistent sputum positivity after two months of antituberculosis treatment

\begin{tabular}{lccc}
\hline Aspect & $\begin{array}{c}\text { Sputum status after two } \\
\text { Negative }(\mathrm{n}=358) \\
(\%)\end{array}$ & $\begin{array}{c}\text { months of treatment } \\
\text { Positive }(\mathrm{n}=55) \\
(\%)\end{array}$ & P-value \\
\hline Chest X-ray lesions involving & 51.4 & 34.5 & \\
$\quad \leq 1$ zone & 48.6 & 65.5 & 0.02 \\
$\quad \begin{array}{l}2 \text { zones } \\
\text { Chest X-ray showing cavities }\end{array}$ & 61.5 & 67.3 & \\
$\quad$ Yes & 38.5 & 32.7 & 0.40 \\
$\quad$ No & & & \\
Chest X-ray showing & 56.4 & 50.0 & \\
$\quad$ Single cavity & 43.6 & 50.0 & \\
$\quad$ Multiple cavities & & & \\
HIV status & 27.7 & 40.0 & \\
$\quad$ Positive & 72.3 & 60.0 & \\
$\quad$ Negative & & & \\
Sputum AFB smear & 57.3 & 36.4 & 0.004 \\
$\quad$ 2+ & 42.7 & 63.6 & \\
3+ & & & \\
\hline
\end{tabular}

Table 3

Logistic regression analysis for factors independently associated with persistent sputum positivity after two months of treatment

\begin{tabular}{lrccc}
\hline Factor & $\beta$-coefficient & P-value & Odds ratio & \multicolumn{1}{c}{ 95\% CI } \\
\hline Age $\geq 40$ years & -0.9992 & 0.003 & 2.716 & $1.412-5.223$ \\
Male sex & 0.7193 & 0.067 & 2.053 & $0.952-4.43$ \\
Alcohol use & 0.5499 & 0.148 & 1.733 & $0.823-3.65$ \\
Tobacco smoking & -0.0460 & 0.910 & 0.955 & $0.431-2.12$ \\
Two or more lung zones affected & 0.6659 & 0.104 & 1.945 & $0.871-4.35$ \\
$\begin{array}{l}\text { Two or more months delay } \\
\text { before diagnosis }\end{array}$ & -0.2291 & 0.558 & 0.795 & $0.369-172$ \\
HIV negative status & -0.6069 & 0.074 & 0.545 & $0.280-1.06$ \\
$\begin{array}{l}\text { Sputum bacillary load at } \\
\text { diagnosis at 3+ }\end{array}$ & 0.6706 & 0.037 & 1.955 & $1.039-3.68$ \\
\hline
\end{tabular}

Table 4

Final treatment outcomes in patients with smear positive pulmonary tuberculosis in relation to sputum smear conversion status at two months $(n=413)$

\begin{tabular}{lcccc}
\hline Final treatment outcomes & \multicolumn{2}{c}{$\begin{array}{l}\text { Sputum smear status after two months of treatment } \\
\end{array}$} & Negative $(\mathrm{n}=358)$ & \multicolumn{2}{c}{ Positive $(\mathrm{n}=55)$} \\
& No. & $(\%)$ & No. & $(\%)$ \\
\hline Cured & 251 & 70.1 & 26 & 47.3 \\
Treatment completed & 43 & 12.0 & 10 & 18.2 \\
Treatment failure & 0 & 00.0 & 01 & 01.8 \\
Defaulted & 54 & 15.1 & 14 & 25.5 \\
Died & 4 & 01.1 & 00 & 00.0 \\
Transferred out & 6 & 01.7 & 04 & 07.2 \\
\hline Total & 358 & 100 & 55 & 100 \\
\hline
\end{tabular}


Table 5

Comparison of final favourable and unfavourable treatment outcomes in 413 patients with smear positive pulmonary tuberculosis in relation to sputum smear conversion status at two months

\begin{tabular}{lccccc} 
Final treatment outcome & \multicolumn{5}{c}{ Sputum smear status after two months of treatment } \\
& $\begin{array}{l}\text { Negative }(\mathrm{n}=358) \\
\text { No. }\end{array}$ & $\begin{array}{c}\text { Positive }(\mathrm{n}=5) \\
\text { No. }\end{array}$ & $(\%)$ & P - value \\
\hline Favourable & 294 & 83.5 & 36 & 70.6 & \\
Unfavourable & 58 & 16.5 & 15 & 29.4 & 0.025 \\
\hline
\end{tabular}

The final treatment outcomes of the 413 patients with smear positive pulmonary tuberculosis in relation to their smear conversion status at the end of two months of treatment is presented in Table 4. Only $36(70.6 \%)$ of the 55 patients with persistent positive sputum smears at the end of two months of treatment as compared to $294(83.5 \%)$ of the 358 patients with negative sputum smears (Table 5 ) had a favourable outcome $(\mathrm{p}=0.025)$.

\section{DISCUSSION}

The search for tools to monitor the treatment of tuberculosis patients and predict its outcome continues. Studies have shown that non conversion of positive smears at the end of two months of treatment is one of the strongest predictors for treatment failure (3-6) although it is not a very reliableindicator because of its low positive predictive value.

Indeed a positive sputum smear for AFB does not permit one to know whether these are still viable bacteria or not after two months of antituberculosis treatment. To assess sputum sterilisation therefore, it is ideal to study cultures for mycobacteria at the end of two months, as microscopy may also show non viable bacilli. However under programme conditions, particularly in most low income countries like Cameroon, cultures for mycobacteria are not available under field conditions. Furthermore, conventional results of culture on media would be available far too late (after more than two months) to be useful. The correlation between sputum AFB smear and culture is generally good (9) although there are reports on the poor correlation of smear and culture during treatment and the unreliability of smear for declaration of failure (10). Studies have however shown that in countries where limited culture and sensitivity studies for mycobacteria are available, sputum microscopy at two months may be a critical determinant of high and low risk patients for treatment failure (3-4). Sputum smear examination may therefore serveas a proxy for culture examination in low income countries under programme conditions, keeping in mind its limitations.

In this study, non conversion of sputum specimens of new smear positive patients with pulmonary tuberculosis after two months of treatment was more likely to be associated with unfavourable outcomes in terms of treatment failure and particularly default from treatment. Our results are in agreement with those of previous studies that have shown that among sputum positive pulmonary tuberculosis patients, persistent sputum positivity at the end of the initial two months of treatment can predict adverse outcomes (3-7). The development of tools which can prospectively identify patients with delayed sputum sterilisation so that they can then be better managed is therefore necessary. Some techniques have been described (11-13) but these are still too sophisticated for routine use even in developed countries. As such, there is a need in developing countries particularly those of sub-Saharan Africa where tuberculosis is highly prevalent that simple predictive factors associated with non conversion of sputum smears after the initial two months of treatment be identified.

In this study we assessed clinical, microbiological and radiological factors in new pulmonary tuberculosis patients receiving treatment under direct supervision by nurses in accordance with routine national tuberculosis control guidelines in a bid to identify simple factors associated with non conversion of positive sputum smears at the end of the initial two months of treatment. Univariate analysis of these factors showed that delays superior to one month before diagnosis, the presence of cavities on chest X-rays of patients as well as an HIV seropositive status were not significantly associated with non conversion of positive sputum smears. Of the factors that showed a significant relationship with persistent sputum positivity after two months of antituberculosis therapy on univariate analysis, only age above or equal to 40 years and numerous bacilli (3+) on pre-treatment sputum smears remained significant independent predictors on multivariate analysis (Table 3 ).

The absence of a significant relationship between cavitary disease and non conversion of positive sputum smears after two months of treatment in our study is not in line with results from other reports (714-15). Cavitary disease has been found by Telzac et al (14) to be independently associated with a greater 
number of days for sputum smear and culture conversion. Hiyama et al (15) also reported in a study looking at factors influencing response to treatment that progressive chest $X$-ray findings were associated with delayed sputum smear conversion. Finally in a study similar to ours, Singla et al (7) in Riyadh, Saudi Arabia reported that the presence of multiple cavitary lesions on chest $\mathrm{X}$-ray were significantly associated with persistent sputum smear positivity.

HIV seropositivity was not associated with the non conversion of positive sputum smears after two months of antituberculosis treatment in our study. Our results are in agreement with those of several studies which have shown that HIV positive patients co-infected with pulmonary tuberculosis do not show delayed sputum conversion $(14,16,17)$. On the contrary it has even been argued by some authors (14) that HIV positive patients may instead have early sputum conversion.

Age above or equal to 40 years was an independent predictor of non-conversion of sputum smears in our smear positive pulmonary tuberculosis patients after theinitial two months of antituberculosis chemotherapy. Singla et al (7) observed in a similar study that patients aged over 60 years had an almost six times greater risk of remaining sputum positive after two months of treatment than patients aged 2140 years, while patients aged 41-60 years were twice as likely to remain sputum positive. Liu et al (16) also reported that the elderly were the least likely to have documented sputum conversion after two months of treatment. The reason why old age should predispose to delayed sputum smear conversion is not exactly known.

In this study, the presence of numerous bacilli (3+) on initial pre-treatment sputum smears was also an independent predictor of non conversion of positive sputum smears after two months of antituberculosis therapy. The direct influence of initial bacillary load on the absence of sputum conversion at two months of therapy has been reported by several authors $(3,5,7,15)$. Rieder (3) observed that in a refugee camp in Thailand, sputum conversion at the end of the two months of directly observed therapy among patients with initial weakly positive sputum was $90.9 \%$ while it was respectively $77.9 \%$ and $61.7 \%$ among patients with initial moderately positive and strongly positive sputum (3). However in an adjusted analysis, he observed that initial smear positivity was not an independent predictor for subsequent positivity at four months or later. Singla et al (7) reported that patients with numerous bacilli on pre-treatment sputum smear examination had an almost six times greater risk of persistent sputum positivity than patients with few bacilli. Meanwhile, Lienhart et al (5) reported sputum conversion at theend of two months in patients with initial sputum smear $1+, 2+, 3+$ to be
$96.2 \%, 85.8 \%$, and $81.8 \%$ respectively. They further reported that treatment failure was associated with increasing bacillary load while the absence of sputum smear conversion after two months was associated with defaulting later during treatment, a result similar to ours.

In conclusion, non conversion of positive sputum smears in new patients with pulmonary tuberculosis in Yaoundé, Cameroon, at the end of two months of treatment is associated with unfavourable treatment outcomes particularly defaulting later during the course of therapy. In this setting, non conversion of sputum smears after the initial two months of antituberculosis treatment is associated with age above or equal to 40 years and the presence of numerous bacilli $(3+)$ on pre-treatment sputum smears. It is therefore recommended that patients with these factors who do not smear convert after two months of antituberculosis treatment in Yaoundé, be given a fully supervised treatment for the entire duration of chemotherapy so as to prevent in particular default from treatment.

\section{REFERENCES}

1. Enarson, D.A., Rieder, H.L., Arnadottir, T. and Trebucq, A. Prise en charge de la tuberculose : guide pour les pays à faibles revenus. 5è éd Paris ; Union Internationale Contre la Tuberculose et les Maladies Respiratoires, 2000.

2. World Health Organisation (WHO). Treatment of tuberculosis. Guidelines for National Programmes. $2^{\text {nd }}$ ed. Geneva: WHO, 1997.

3. Rieder,H.L.Sputum smear conversion during directly observed treatment for tuberculosis. Tubercle. Lung. Dis. 1996; 77:124-129.

4. Feng-Zeng, Z., Levy, M.H. and Sumin, W. Sputum microscopy results at two months predict outcome of tuberculosis treatment. Int. J. Tuberc. Lung Dis. 1997; 1:570-572.

5. Lienhart, C., Manneh, K., Bouchier, V., et al Factors determining the outcome of treatment of adult smearpositive cases in the Gambia. Int. J. Tuberc. Lung Dis. 1998; 2:712-718.

6. Salaniponi, F.M., Christensen, J.J., Gausi, F., et al. Sputum smear status at two months and subsequent treatment outcome in new patients with smear positive pulmonary tuberculosis. Int. J. Tuberc. Lung Dis. 1999; 3:1047-1048.

7. Singla, R., Osman, M.M., Khan, N., et al. Factors predicting persistent sputum smear positivity among pulmonary tuberculosis patients 2 months after treatment. Int. J. Tuberc. Lung Dis. 2003; 7:58-64.

8. Chavez, Pachas, A.M., Blank, R., Smith, Fawzi, M.C., et al. Identifying early treatment failure on category 1 therapy for pulmonary tuberculosis in Lima Ciudad, Peru. Int. J. Tuberc. Lung Dis. 2004; 8:52-58.

9. Hobby, G.I., Holman, A.P., Iseman, M.D. and Jones J.M. Enumeration of tubercle bacilli in sputum of 
patients with pulmonary tuberculosis. Antimicrob. Agents Chemother. 1973; 4:94-104.

10. Vidal, R., Martin-Casabonan, N., Juan, A., Falgueras, T. and Miravitles, M. Incidence and significanceof acid-fast bacilli in sputum smears at the end of antituberculous treatment. Chest. 1996; 109:1562-1565.

11. Epstein, M. D., Schluger, N. W., Davidow, A. L., et al. Time to detection of $M$. tuberculosis in sputum culture correlates with outcomein patients receiving treatment for pulmonary tuberculosis. Chest. 1997; 113:379-386.

12. Wallis, R.S., Perkins, M.D., Philips, M.et al.Predicting the outcome of therapy for pulmonary tuberculosis. Am. J. Respir. Crit. Care Med. 2000; 161:1076-1080.

13. Wallis, R.S., Perkins, M.D., Philips, M., et al.Induction of antigen 85 complex of M. tuberculosis in sputum: a determinant of outcome of therapy in pulmonary tuberculosis. J. Infect. Dis. 1998; 178:1115-1121.
14. Telzac, E.E., Fazal, B.A., Pollard, C.L., et al. Factors influencing time to sputum conversion among patients with smear positive pulmonary tuberculosis. Clin. Infect. Dis. 1997; 25: 666-670.

15. Hiyama, J., Marukawa, M., Shiota, Y., et al. Factors influencing response to treatment of pulmonary tuberculosis. Acta. Med. Okayama. 2000; 54:139-145.

16. Liu, Z., Shilkret, K.L. and Elis, H.M. Predictors of sputum culture conversion among patients with tuberculosis in the era of tuberculosis resurgence. Arch Intern. Med. 1999;159:1110-1116.

17. Bwire, R., Borgdorff M.W., Sticht-Groh, V. et al. Tuberculosis chemotherapy and sputum conversion among HIV seropositive and HIV seronegative patients in south-eastern Uganda. East. Afr. Med. J. 1999;76:307-313. 